\title{
EL TLC PERU-ESTADOS UNIDOS: REINSERCIÓN ECONÓMICA INTERNACIONAL O SUBORDINACIÓN NACIONAL
}

Francisca Bouby Tolentino (*)

E-mail:f_bouby@yahoo.com

\begin{abstract}
RESUMEN
El TLC entre Perú y Estados Unidos tiene como objetivos tanto la consolidación y ampliación de los beneficios temporales establecidos para determinados productos con la ATPA y su posterior ratificación con la ATPDEA, así como intensificar las políticas de ajuste estructural. Todo esto pone en cuestionamiento las funciones mismas del Estado, en su papel de conductor de acciones de coordinación y promoción de las fuerzas productivas nacionales, acciones conducentes a superar las barreras existentes para alcanzar niveles de productividad y competitividad necesarios para hacer frente a la alta competencia que significa un TLC con una economía desarrollada como Estados Unidos y lograr una reinserción activa a la economía global, generando un crecimiento endógeno. La alternativa es dejarse llevar por las fuerzas de atracción en un contexto de laissez faire que conduciría a una reinserción pasiva, lo cual generaría mayor polarización entre los miembros de la sociedad peruana.
\end{abstract}

Palabras Claves: Productividad, competitividad, reinserción, Estado.

\begin{abstract}
The Free Trade Agreement (FTA) between Peru and the United States of America has as objectives the consolidation and the enlargement of the temporary benefits established, for certain products, with the ATPA and the further ratification with the ATPDEA and also to intensify a structural adjustment policies. These issues set in question the functions of the State, in its role of leading the actions of coordination and promotion of the national productive forces, leading actions to overcome the existing barriers in order to reach productivity and competitve levels needed to face up the high competition that signifies a FTA with a developed economy as the USA and to achieve an active reinsertion in the global economy generating an endogenous growing. The alternative is to let be taken to the forces of attraction in a context of laissez-faire that would lead to a passive reinsertion generating bigger polarization among the members of the Peruvian society.
\end{abstract}

Key Words: Productivity, competitiveness, reinsertion, State.

(*) Doctora en Economía. Magister en Economía con mención en Política Económica. Profesora Principal de la Facultad de Ciencias Administrativas (UNMSM). 


\section{INTRODUCCION}

El presente trabajo tiene por objetivo analizar las posibilidades de desarrollo de la economía peruana a partir de su reinserción a la economía global, en el marco de un proceso de integración económica a una de las economías más grandes del mundo como es la estadounidense, en el contexto de un Tratado de Libre Comercio (TLC). Asimismo, analizar los principales temas vulnerables que limitan las posibilidades de una reinserción activa para generar desarrollo económico, lo cual implicaría una mayor polarización en la sociedad peruana.

En tal sentido, en el primer bloque se esboza el marco teórico, donde se destacan conceptos como el de reinserción activa o pasiva de las economías en desarrollo a la economía globalizada, y la tendencia de las economías latinoamericanas de dar una respuesta pasiva, generando con ello una mayor polarización en la sociedad.

En el segundo bloque el análisis se centra en el Tratado de Libre Comercio del Perú con Estados Unidos, destacándose en el acápite los objetivos económicos de dicho Tratado; las principales asimetrías entre ambos países miembros del Tratado y el perfil económico del Perú, destacando el origen y destino de la inversión extranjera directa (IED); la composición de las exportaciones; los indicadores de estabilidad macroeconómica y la situación del empleo y desempleo en el Perú.

En el tercer bloque se destacan los temas vulnerables de la economía peruana para el aprovechamiento de las oportunidades aperturadas con el TLC, donde se señalan las limitaciones existentes en tecnología y en el mercado laboral para alcanzar indicadores de productividad y competitividad frente a la alta competencia que se generaría ante la puesta en marcha del Tratado, toda vez que la calidad del mercado laboral define la competitividad de las economías.

\section{MARCO TEÓRICO}

La dinámica mundial de las últimas décadas ha modificado los referentes que explicaban la integración económica internacional entre países desarrollados y en desarrollo. El surgimiento de un nuevo paradigma tecnológico y el cambio en la organización del espacio mundial, han determinado el agotamiento de los viejos estilos de integración y la aparición de nuevas formas de vinculación entre los países en el capitalismo global, redefiniéndose de este modo las posibilidades de desarrollo económico, crecientemente dependiente de la articulación de las economías nacionales a la producción mundial integrada $o$ redes mundiales ${ }^{1}$.

Las cadenas de producción mundiales se organizan principalmente sobre bases flexibles ${ }^{2}$, siendo determinante la evolución de la empresa subcontratista para comprender la constitución actual de las redes productivas globales ${ }^{3}$.

Actualmente existe un amplio debate relacionado con las posibilidades de desarrollo económico que se apertura a partir de dicha integración o articulación a la producción global, frente a la alternativa de la marginación o exclusión social.

Para evaluar el proceso de reinserción internacional de los países en desarrollo, específicamente de los países latinoamericanos, en un contexto en el que prevalece el libre comercio y el Estado a favor de éste, se requiere de un marco conceptual que facilite la discusión de las nuevas posibilidades históricas de desarrollo económico.

En primer lugar, hablar de reinserción internacional implica entender que existen cambios en la estructura de la economía global y que a su vez éstos cambios provocan un conjunto de repercusiones en los países en desarrollo, y solo aquellos países que orienten sus esfuerzos a procesos internos de reestructuración de sus economías, destinados a aprovechar las oportunidades y afrontar los retos mundiales, podrán hallar una nueva ubicación en la división internacional del trabajo.

En segundo lugar, se requiere de una respuesta activa frente a los cambios, con acciones de coordinación emprendidas por el Estado para movilizar a los agentes productivos nacionales, como empresarios y trabajadores, con el fin de resolver problemas de asignación de recursos que no logran hacer las fuerzas del mercado. Una respuesta activa implica también un proceso de desarrollo de la capacidad de los agentes productivos de asimilar conocimientos tecnológicos para reproducir y luego innovar los sistemas de producción; aprendizaje que al hacerse extensivo al conjunto de la sociedad, puede con- 
ducir a la inserción de los grupos sociales en los sistemas socio-económicos e institucionales, de acuerdo al nuevo paradigma tecnológico ${ }^{4}$.

En contraste, la falta de políticas estatales de coordinación y aprendizaje (que podemos entender como una respuesta pasiva), inmoviliza a los agentes productivos nacionales, dejando enteramente al mercado la solución del problema de asignación de recursos, limitándose de este modo las posibilidades de dinamismo económico, lo cual conduce a profundizar las desigualdades sociales, sectoriales y regionales.

A fin de complementar el análisis sobre las posibilidades de desarrollo en el mundo global actual, se sintetizan los resultados del debate aperturado en torno a la búsqueda de mejores posiciones en las cadenas productivas globales, y en relación al papel que juega el Estado en los países en desarrollo.

En este debate sobresalen dos aspectos fundamentales. Primero, el reconocimiento de la nueva teoría del comercio internacional, que sostiene que el beneficio mutuo deja de ser el resultado inevitable del libre comercio, aceptándose con ello la existencia de relaciones asimétricas en el intercambio comercial. ${ }^{5}$ Segundo, dada la creciente brecha entre países desarrollados y subdesarrollados, una integración activa a la economía global no podrá generarse espontáneamente. Esta requiere de una vasta acción promotora e intervención sistemática del Estado en coordinación con las principales fuerzas productivas de la economía pero con una visión de largo plazo, con políticas de aplicación sectorial selectiva y con un sólido marco disciplinario anticorrupción. De lo contrario, una integración pasiva que implica dejarse llevar por las fuerzas de atracción en un contexto de laissez faire impuesto, conduciría a una creciente subordinación nacional ${ }^{6}$.

En consecuencia, en el debate actual sobre las posibilidades de desarrollo en el sistema capitalista, dados los factores externos, el análisis se centra en los factores internos socioinstitucionales y socio-culturales para determinar la capacidad de asimilación real de los avances tecnológicos, lo cual implica el desarrollo de dos aspectos fundamentales, la coordinación y el aprendizaje ${ }^{7}$.

En tercer lugar, cabe resaltar la importancia de las condiciones endógenas de crecimien- to, planteadas por las diferentes corrientes económicas, de las cuales se desprende la necesidad de replantear las estrategias de liberalización y de implementación de políticas sectoriales de educación, capacitación, empleo, tributarias, reforma del Estado, etc., para integrar las economías nacionales y/o regionales al mercado mundial. Se destacan asimismo aquellos planteamientos que van más allá de la crítica al modelo IOE, desarrollando conceptos para distinguir cambios en los procesos de producción y de trabajo, sustentabilidad económica, ventajas absolutas y encadenamientos mercantiles globales; elementos cruciales para la viabilidad de las economías en el contexto actual de la economía globalizada ${ }^{8}$.

\section{EL TLC PERÚ - ESTADOS UNIDOS}

\section{Objetivos}

El 18 de mayo de 2004 se dio inicio a las negociaciones de un Tratado de Libre Comercio (TLC) entre Perú y Estados Unidos. Luego de trece rondas de negociación, ambos países acordaron un texto final el 7 de diciembre de 2005 en Washington, D.C.

Uno de los principales objetivos económicos del TLC es la consolidación y ampliación de los beneficios temporales obtenidos para los productos agrícolas con la Ley de Preferencias Arancelarias Andinas (ATPA según sus siglas en inglés) y su posterior ratificación por la Ley de Promoción Comercial Andina y Erradicación de la Droga (ATPDEA según sus siglas en inglés), el cual vence el 31 de diciembre del presente año.

Específicamente con el TLC se busca consolidar el aprovechamiento de nuestro potencial de la agricultura no tradicional relacionado con verduras, hortalizas y frutas frescas. En el 2003, Perú se había posicionado como el cuarto proveedor de vegetales frescos a Estados Unidos, muy por encima de países como Israel, China, Chile, siendo tan sólo superados por los socios del Tratado de Libre Comercio de América del Norte (TLCAN) y Holanda. Sin embargo, Estados Unidos no renuncia a la política de subsidios para la agricultura, ni a otras medidas proteccionistas consideradas necesarias, por lo que los productos denominados sensibles tales como azúcar, carnes, arroz y leche, y específicamente el algodón, maíz y trigo, podrían verse afecta- 
dos por la entrada de productos importados de Estados Unidos.

El TLC también busca intensificar aquellos aspectos fundamentales que orientaron las políticas de ajuste estructural de la globalización neoliberal en las dos últimas décadas, tales como la liberalización, la desregulación y la privatización. Es decir, busca profundizar la liberalización de los mercados de bienes, servicios, inversiones y derechos de propiedad intelectual, además de eliminar los aranceles a la importación y los subsidios a la producción interna; asimismo, limitar las funciones del Estado en su papel de regulador y conductor de una estrategia de desarrollo nacional, dejándose plena libertad a las empresas transnacionales, como conductores del proceso de globalización e integración.

Estas políticas vienen subordinando los intereses de los estados nacionales a las institu- ciones supranacionales, alentando la alta competencia para atraer inversiones a nuestras economías en base a la desregulación del mercado laboral y al deterioro del medio ambiente, por lo que el Esta-do peruano debe propiciar una respuesta a estos procesos, asumiendo una función promotora y coordinadora para una reinserción activa al mercado global, con objetivos concretos en base a un modelo de desarrollo nacional, a fin de evitar una mayor polarización de la sociedad.

\section{Principales asimetrías entre Perú y Estados Unidos}

Los principales indicadores socio-económicos del Perú y Estados Unidos reflejan las enormes asimetrías existentes entre ambas economías. En el Cuadro 1 se muestra el perfil socio-económico de cada país miembro del TLC,

Cuadro 1

Asimetrías Perú - Estados Unidos

\begin{tabular}{|c|c|c|}
\hline Área comparativa & Estados Unidos & Perú \\
\hline PBI - Per Cápita & $\begin{array}{c}\$ 42,000(2005 \text { est.) } \\
\text { PBI }\end{array}$ & $\begin{array}{c}\$ 6,100 \text { (2005 est.) } \\
\$ 169,5 \text { mil millones } \\
\text { (2005 est.) }\end{array}$ \\
\hline Uso de las tierras & Región cultivable: $18,01 \%$ & Región cultivable: $2,88 \%$ \\
\hline Población & $298,444,215$ (julio 2006 est.) & $28,302,603$ (julio 2006 est.) \\
\hline $\begin{array}{c}\text { Tasa de Mortalidad } \\
\text { Infantil }\end{array}$ & $\begin{array}{c}\text { Total: } 6,43 \text { muertes } / 1,000 \\
\text { partos que viven }\end{array}$ & $\begin{array}{c}\text { Total: } 30,94 \text { muertes } / 1,000 \\
\text { partos que viven }\end{array}$ \\
\hline $\begin{array}{c}\text { Tasa de Desempleo: } \\
\text { Población debajo del } \\
\text { umbral de pobreza }\end{array}$ & $5,1 \%$ (2005 est.) & $\begin{array}{c}8,7 \% \text { en Lima Metropolitana } \\
(2005 \text { est.) }\end{array}$ \\
\hline
\end{tabular}

\begin{tabular}{|c|c|c|}
\hline \multicolumn{3}{|c|}{ Transporte } \\
\hline Aeropuertos & $14,893(2005)$ & $246(2005)$ \\
\hline Puertos & $\begin{array}{c}\text { Corpus Christi, Duluth, Hampton Roads, } \\
\text { Houston, Long Beach, Los Angeles, } \\
\text { Nueva Orleans, Nueva York, Filadelfia, } \\
\text { Tampa, Texas City } \\
\text { terminales } \\
\text { Nota:13 puertos al norte de Nueva } \\
\text { Orleans (South Luisiana Puertos) sobre el } \\
\text { Mississippi River maneja 290,000,000 } \\
\text { tonelada de carga anualmente }\end{array}$ & $\begin{array}{c}\text { Callao, Iquitos, } \\
\text { Yatarani, Pucallpa, } \\
\text { Pucallpa, y } \\
\text { Yurimaguas }\end{array}$ \\
\hline
\end{tabular}

\begin{tabular}{|l|l|l|}
\hline \multicolumn{3}{|c|}{ Comunicaciones } \\
\hline Teléfonos - líneas principales en uso & 268 millones (2003) & $2,049,800$ (2004) \\
\hline
\end{tabular}

Fuente: CIA - The World Factbook, United States; mayo, 2006 
donde se destacan aspectos favorables, como el tamaño del mercado de Estados Unidos, que podría favorecer la demanda potencial de nuestros productos no tradicionales, así como aspectos no tan favorables como el índice de uso de zonas de cultivo que muestra a Estados Unidos con una utilización del $18.01 \%$ de su región cultivable, mientras que Perú sólo usa el 2.88\%, mostrando una de nuestras debilidades que es resultado de la falta de preocupación por desarrollar zonas que no están siendo utilizadas.

Igualmente los indicadores económicos que demuestran debilidades son aquellos relacionados con el desarrollo de comunicaciones y transporte. Entre estos últimos, aquellos vinculados con la infraestructura portuaria y aeroportuaria, los cuales presionan sobre los costos y éstos inciden a su vez en los indicadores de productividad y competitividad de nuestras empresas y productos exportables.

De otro lado, las enormes diferencias que muestran los indicadores socioeconómicos, tales como tasa de mortalidad infantil, tasa de desempleo, PBI per cápita y la población por debajo del umbral de pobreza (54\% en el caso de Perú), demuestran la amplia brecha existente entre un país desarrollado como Estados Unidos y uno subdesarrollado como Perú, pudiendo acentuarse estas diferencias a medida que avanza el proceso de globalización e integración, si es que nuestra economía responde de manera pasiva a estos procesos.

\section{PERFIL ECONÓMICO DEL PERÚ}

\section{Origen y destino de la Inversión Extranjera Directa (IED)}

La IED proviene, principalmente, de tres países: España, Estados Unidos y Reino Unido. En conjunto, la IED proveniente de estos tres países representa el $64.5 \%$ de la IED total realizada en el Perú durante el año 2005. Si bien Estados Unidos sigue siendo una importante fuente de IED (15.8\%), en los últimos años, ha sido superado por España (33.1\%). (Cuadro 2).

En cuanto al destino de las inversiones, el sector comunicaciones ha pasado a ser el principal destinatario (34.7\%). Si bien la minería e industria siguen siendo importantes receptores de IED ( $14.8 \%$ y $15.3 \%$ respectivamente), han entrado a tallar los sectores finanzas y energía (12.5\% y $11.5 \%$ respectivamente). (Ver Cuadro 3 )

\section{Cuadro 2}

Fuentes de la IED en el Perú

(En millones de dólares)

\begin{tabular}{|l|r|r|}
\hline \multicolumn{1}{|c|}{ PAÍS 1/ } & \multicolumn{1}{c|}{$\mathbf{2 0 0 5}^{*}$} & $\boldsymbol{\%}$ \\
\hline ESPAÑ $\Lambda$ & $4,726.00$ & 33.00 \\
\hline EE.UU. & $2,262.30$ & 15.84 \\
\hline REINO UNIDO & $2,227.40$ & 15.60 \\
\hline COLOMBIA & 849.5 & 5.95 \\
\hline PAISES BAJOS & 779.2 & 5.46 \\
\hline PANAMA & 602.8 & 4.22 \\
\hline CHILE & 490.5 & 3.44 \\
\hline MEXICO & 431.6 & 3.02 \\
\hline OTROS & $1,908.9$ & 13.37 \\
\hline I.E.D & $14,278.20$ & 100.00 \\
\hline
\end{tabular}

1/ País declarado como domicilio en el registro de la inversión. Fuente: PROINVERSIÓN, CONASEV y Registros Públicos al 31 de diciembre del 2005.

\section{Cuadro 3}

IED según sector de destino (En millones de dólares)

\begin{tabular}{|l|r|r|}
\hline \multicolumn{1}{|c|}{ SECTOR } & \multicolumn{1}{c|}{$\mathbf{2 0 0 5 *}$} & \multicolumn{1}{c|}{$\%$} \\
\hline Agricultura & 44.4 & 0.31 \\
\hline Comercio & 600.9 & 4.21 \\
\hline Comunicaciones & $4,953.2$ & 34.69 \\
\hline Construcción & 81.5 & 0.57 \\
\hline Energía & $1,637.6$ & 11.47 \\
\hline Finanzas & $1,787.4$ & 12.52 \\
\hline Industria & $2,190.4$ & 15.34 \\
\hline Minería & $2,115.8$ & 14.82 \\
\hline Pesca & 0.6 & 0.00 \\
\hline Petróleo & 207.0 & 1.46 \\
\hline Scrvicios & 265.4 & 1.86 \\
\hline Silvicultura & 1.2 & 0.01 \\
\hline Transporte & 313.4 & 2.20 \\
\hline Turismo & 62.1 & 0.43 \\
\hline Vivienda & 16.1 & 0.11 \\
\hline Total Acum. & $14,278.1$ & 100.00 \\
\hline
\end{tabular}

Fuente: *PROINVERSIÓN, CONASEV y Registros Públicos al 31 de diciembre del 2005.

Las inversiones más importantes provienen de privatizaciones realizadas en comunicaciones. En energía resaltan las privatizaciones de varias generadoras y distribuidoras de energía. En finanzas, destaca la privatización del Banco Continental. En manufactura la inversión se ha dado, principalmente, bajo la modalidad de compra o adquisición, parcial o total.

\section{Composición de las exportaciones}

Las exportaciones peruanas se basan principalmente en recursos naturales debido a que 
en la economía no se han generado condiciones básicas para una industrialización orientada hacia las exportaciones. Aunque el crecimiento de las exportaciones durante los últimos cinco años ha sido favorable, con una balanza comercial positiva a partir de 2002 (Ver Gráfico 1) y una diversificación de las exportaciones no tradicionales (Ver Gráfico 2), este crecimiento exportador se ha basado fundamentalmente en las exportaciones tradicionales (Ver Gráfico 3), lo que hace que la economía peruana sea muy sensible a las variaciones de precios internacionales de los commodities.

\section{Gráfico 1}

Perú: Balanza Comercial Total

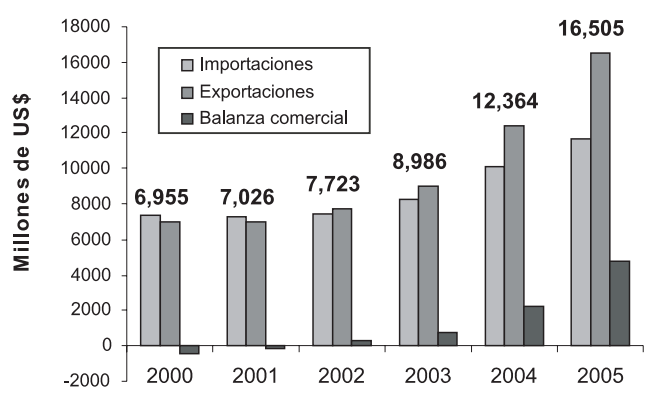

Gráfico 2

Perú: Exportaciones No Tradicionales (En millones de dólares)

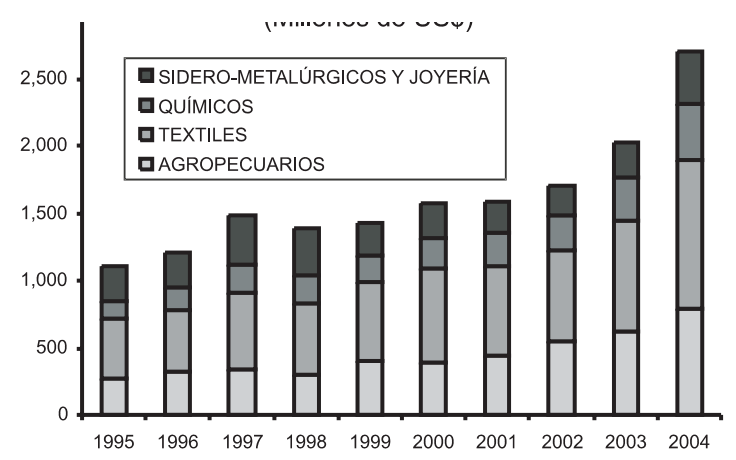

Gráfico 3

Perú: Composición de las exportaciones (En millones de dólares)

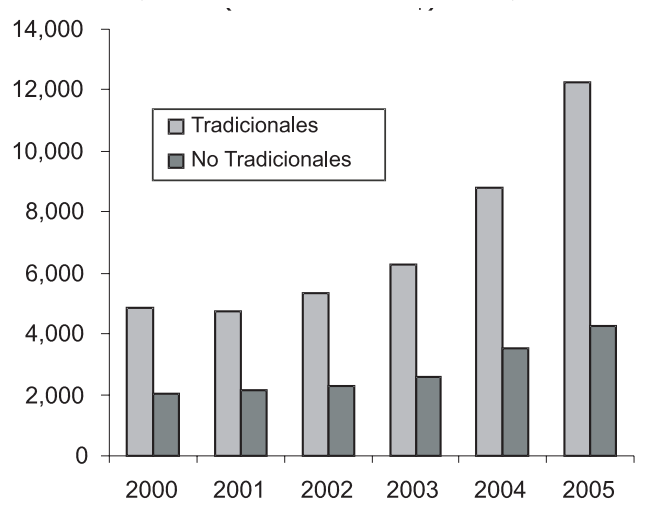

Indicadores de estabilidad macroeconómica en el Perú

Los diferentes indicadores macroeconómicos, tales como nivel de inflación, tipo de cambio, reservas internacionales netas, PBI, tasa de interés, inflación, riesgo-país, entre otros, muestran que la economía peruana está en crecimiento y con estabilidad macroeconómica.

En relación al nivel de inflación, el Perú ha logrado mantener bajos niveles de inflación en los últimos años. La inflación promedio durante el periodo 2002-2005 fue de 2.2\% (Gráfico 4). El tipo de cambio, particularmente estable, ha mostrado una ligera tendencia decreciente que contrarresta ligeramente la sustancial mejora de los términos de intercambio con el exterior durante los últimos años. Tanto el tipo de cambio real como el tipo de cambio nominal experimentaron similar comportamiento desde 1996 hasta el 2006. A enero del presente año, el tipo de cambio nominal representó 3.40 nuevos soles por cada dólar, mientras que el tipo de cambio real representó aproximadamente 3.25 nuevos soles por cada dólar (Gráfico 5).

\section{Gráfico 4}

Inflación 1995-2005

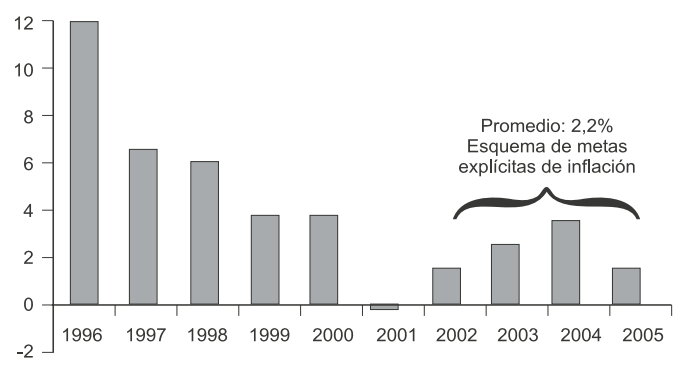

Gráfico 5

Tipo de Cambio Nominal y Real Enero 1995 - Enero 2006

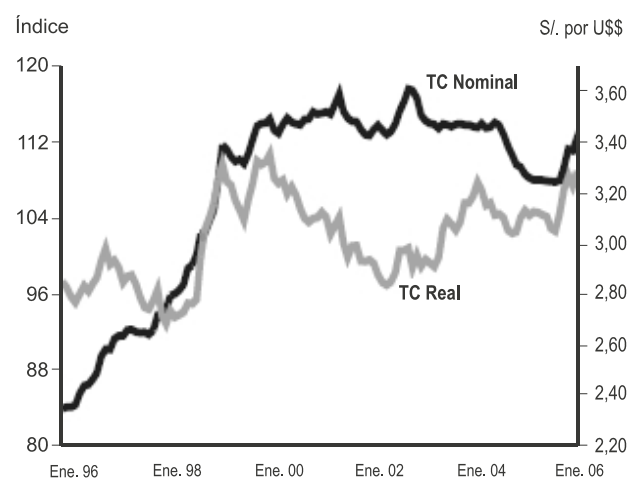


La estabilidad financiera obedece en parte a las altas reservas internacionales netas (RIN) que mantiene el Banco Central de Reserva del Perú (BCRP). Las reservas internacionales han crecido significativamente desde enero del 2004 a mayo del 2006, alcanzando los 14,504 millones de dólares (Ver Gráfico 6). El sostenido aumento de dichas reservas se debe principalmente al desempeño superavitario de la Balanza Comercial (Volver al Gráfico 1).

\section{Gráfico 6}

Reservas Internacionales Netas y Posición de Cambio del BCRP (Ene.2004-May.2006)

(En millones de dólares)

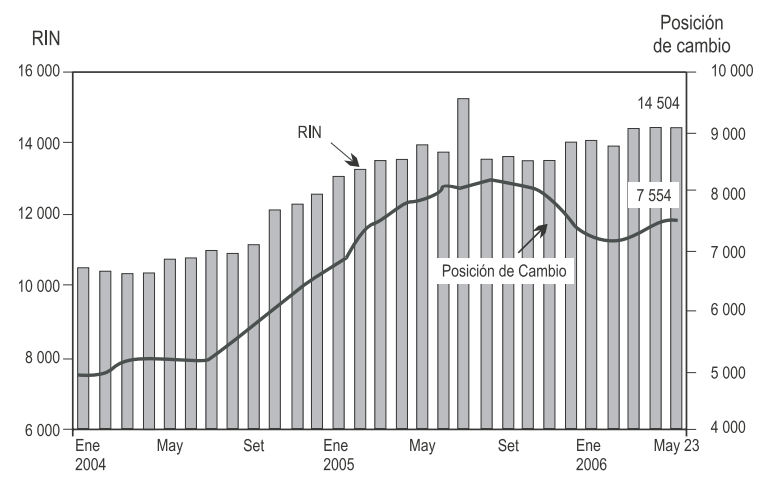

Fuente: BCRP

En relación al indicador de riesgo país, éste se encuentra entre los más bajos de la región latinoamericana (Gráfico 7).

\section{Gráfico 7}

Riesgo País Perú Vs Riesgo Latinoamérica (EMBI+) (En puntos básicos)

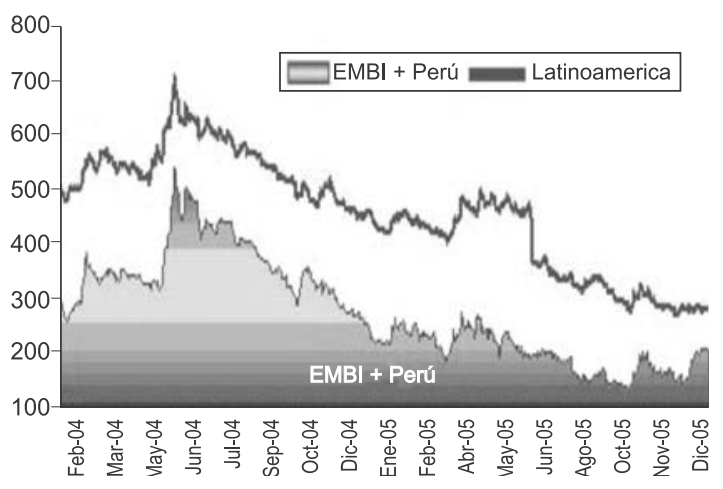

Fuente: Bloomberg

Los indicadores económicos mostrados en los cuadros anteriores reflejan una estabilidad macroeconómica que no se ha visto reflejada positivamente en términos de empleo y condiciones de vida favorables a la población, por lo que el debate desde los noventa va más allá de la estabilidad macroeconómica, incluyendo temas como el combate a la corrupción, la pobreza y el cuestionamiento al Fondo Monetario Internacional (FMI).

Para algunos analistas la economía peruana debería crecer a tasas superiores al promedio observado durante los últimos años (4.5\%) (ver Gráfico 8), a fin de tener resultados importantes en el ingreso real per cápita y con ello reducir el nivel de pobreza en el que se haya postrada más de la mitad de la población.

\section{Gráfico 8}

Evolución mensual del PBI 2004-2006

(Variación \% mensual)

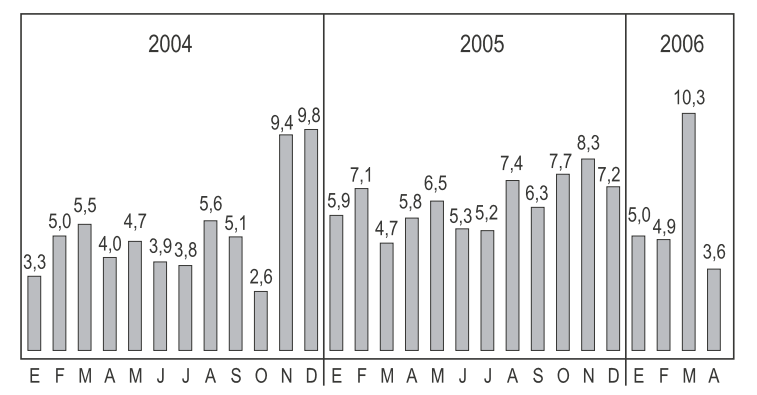

Fuente: INEI

Sin embargo, para crecer sostenidamente con miras a satisfacer las necesidades del mercado interno y orientarse al mercado externo y tener estabilidad macroeconómica, se debe cumplir con condiciones de productividad y competitividad, lo que a su vez implica superar los cuellos de botella existentes, los cuales hacen de la economía altamente vulnerable a los shocks internacionales. Además existe un profundo problema pendiente de redistribución del ingreso.

\section{Situación del empleo y desempleo en el Perú}

El alto desempleo existente en la economía nacional es un factor determinante en la reducción de la demanda interna de productos nacionales, y la consecuente depresión de la industria a nivel nacional.

Al mismo tiempo, la menor producción nacional, ajustada a la menor demanda interna, genera despidos y desempleo, porque la demanda de empleo también se reduce. Esta situación se refleja en las altas tasas de desempleo exis- 
tentes a nivel de las principales ciudades ubicadas al interior del país, como son Chimbote (13.4\%), la Provincia Constitucional del Callao (13.1\%), Arequipa (12.7\%), Cajamarca (12.2\%),
Huancavelica (13.2\%), Cuzco (11.8), Huancayo (11.2\%), Provincia de Lima (11.2\%), Moquegua (10.4\%), Pasco (10.0\%), Tacna (11.0\%), entre otras. (Ver Cuadro 4).

\section{Cuadro 4}

Indicadores de niveles de empleo por ciudades

Año 2005 (\%)

\begin{tabular}{|l|c|c|c|c|}
\hline \multicolumn{1}{|c|}{ Ciudades } & Desempleo & Subempleo & $\begin{array}{c}\text { Adecuadamente } \\
\text { empleados }\end{array}$ & Total \\
\hline Chachapoyas & 6.9 & 52.1 & 41.0 & 100.0 \\
\hline Huaráz & 11.8 & 35.3 & 52.7 & 100.0 \\
\hline Chimbote & 13.4 & 45.4 & 41.2 & 100.0 \\
\hline Abancay & 7.9 & 38.7 & 53.3 & 100.0 \\
\hline Arequipa & 12.7 & 50.1 & 37.2 & 100.0 \\
\hline Ayacucho & 8.4 & 48.1 & 43.5 & 100.0 \\
\hline Cajamarca & 12.8 & 37.5 & 49.7 & 100.0 \\
\hline Cuzco & 11.8 & 44.4 & 43.8 & 100.0 \\
\hline Huancavelica & 13.9 & 42.7 & 43.4 & 100.0 \\
\hline Huánuco & 8.5 & 40.8 & 50.6 & 100.0 \\
\hline Ica & 10.1 & 41.5 & 48.4 & 100.0 \\
\hline Huancayo & 11.3 & 48.4 & 40.3 & 100.0 \\
\hline Trujillo & 8.5 & 44.3 & 47.2 & 100.0 \\
\hline Chiclayo & 8.9 & 48.9 & 42.2 & 100.0 \\
\hline Lima Metropolitana & 11.4 & 40.9 & 47.7 & 100.0 \\
\hline Prov. Const. Callao & 13.1 & 39.4 & 47.4 & 100.0 \\
\hline Prov. de Lima & 11.2 & 41.4 & 47.7 & 100.0 \\
\hline Iquitos & 8.0 & 8.9 & 44.1 & 100.0 \\
\hline Puerto Maldonado & 9.3 & 38.8 & 52.0 & 100.0 \\
\hline Moquegua & 10.4 & 42.6 & 47.0 & 100.0 \\
\hline Cerro de Pasco & 10.0 & 33.9 & 58.1 & 100.0 \\
\hline Piura & 9.3 & 47.0 & 43.7 & 00.0 \\
\hline Puno & 8.9 & 48.7 & 48.4 & 100.0 \\
\hline Tacna & 11.0 & 46.6 & 42.4 & 100.0 \\
\hline Tumbes & 41.2 & 41.0 & 100.0 \\
\hline
\end{tabular}

Fuente: MTPE, Programa de Estadísticas y Estudios Laborales.

PRINCIPALES TEMAS VULNERABLES PARA EL APROVECHAMIENTO DE LA OPORTUNIDADES APERTURADAS CON EL TLC

De acuerdo a la discusión teórica expuesta en el primer acápite de este trabajo, las posibilidades de una reinserción activa de la economía peruana al mercado internacional permitirían que aprovechemos mejor las ventajas tecnológicas y comerciales que se generan con la globalización e integración económica (TLC).

Sin embargo, las condiciones de estabilidad macroeconómica no son suficientes. Existen debilidades en nuestra economía, que se muestra vulnerable frente a los shocks del mercado internacional, y que se constituyen en barreras para el desarrollo de la competitividad, basada en el aumento de la productividad de los factores de la producción, lo cual se refleja en indicadores, tales como, el Índice de Competitividad para el Crecimiento, donde el Perú aparece en el puesto 68 de 117 países; el Índice de Competitividad Empresarial, donde nos encontramos en el puesto 81 de 110 países, y en el Índice de Competitividad Global, donde ocupamos el puesto 77 de 117 países9.

Entre las principales barreras para el desarrollo de la competitividad se encuentran los aspectos relacionados con institucionalidad, infraestructura, mercado laboral, descentralización, entre otros.

Con relación a la institucionalidad, la poca efectividad del aparato estatal a nivel de gobierno, reduce la competitividad. La reinserción de la economía peruana en la economía globalizada 
exige un nuevo tipo de Estado, que debe ser promotor, coordinador, al servicio de la sociedad civil y que promueva el desarrollo empresarial. Se necesita un Poder Judicial transparente en la lucha contra la corrupción, la que resta competitividad a la actividad empresarial.

El nivel de articulación empresarial es muy bajo debido al alto grado de fragmentación en este sector. El nivel de interacción entre los agentes públicos y privados es bajo, y existen muy pocos instrumentos de promoción por parte del Estado que incentiven la consolidación de sistemas empresariales articulados. Se carece de una cultura de innovación y de desarrollo tecnológico, lo que ubica a nuestro país en los peores puestos en estos dos aspectos ${ }^{10}$.

\section{Gráfico 9}

Indicadores de eficiencia en infraestructura portuaria $(7=$ muy bueno $/ 1=$ muy malo $)$

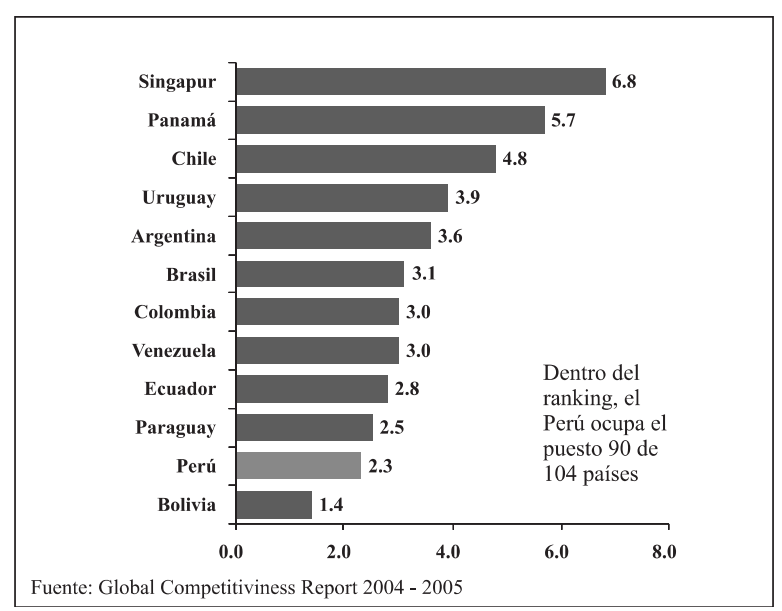

En relación a la infraestructura, ocupamos uno de los lugares más bajos de Latinoamérica respecto a la eficiencia y calidad de la infraestructura y facilidades portuarias. Tenemos costos logísticos muy altos que presionan sobre los costos empresariales, restando competitividad. Así, dentro del ranking de Indice de Eficiencia en Infraestructura, (2004 y 2005), el Perú ocupa el puesto 90 de 104 países (Gráficos 9 y 10).

Uno de los temas que reflejan la vulnerabilidad de nuestra economía es la centralización del PBI (Ver Gráfico 11). Por ejemplo, el 68\% del PBI se halla en concentrado en los cinco primeros departamentos como son, Lima, La Libertad, Arequipa, Junín y Lambayeque. Sólamente Lima centraliza el $46 \%$, mientras que los cinco

\section{Gráfico 10}

Callao - Facturación, sobrecostos e inversión requerida en el corto plazo

(En millones de dólares)

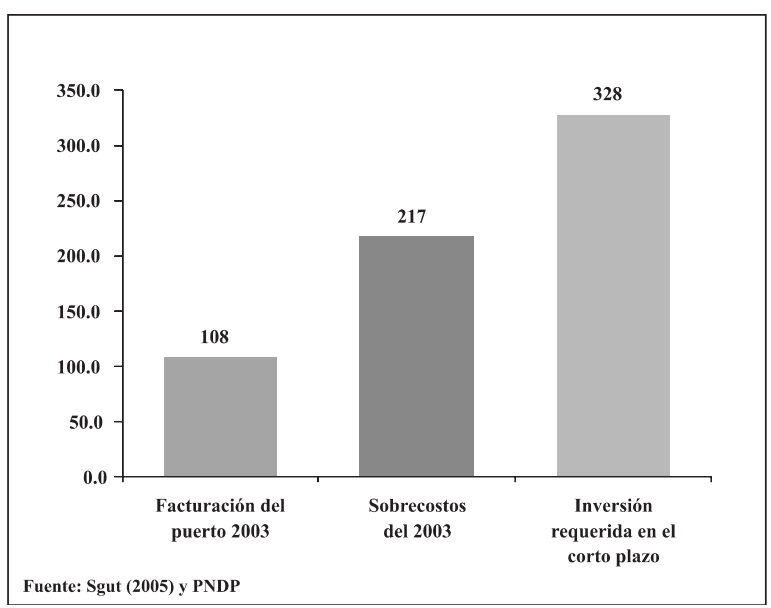

Cuadro 5

Centralización del PBI por Departamentos

\begin{tabular}{|c|c|c|}
\hline Departamento & $\%$ del PBI & \\
\hline $\begin{array}{l}\text { Lima } \\
\text { Arequipa } \\
\text { La Libertad } \\
\text { Junín } \\
\text { Lambayeque } \\
\text { Otros } \\
---------- \\
\text { Huancavelica } \\
\text { Pasco } \\
\text { Apurimac } \\
\text { Tumbes } \\
\text { Madre de Dios }\end{array}$ & $\begin{array}{c}46.30 \\
6.79 \\
5.87 \\
4.64 \\
4.02 \\
------ \\
-29.59 \\
0.87-- \\
0.65 \\
0.62 \\
0.45 \\
0.20\end{array}$ & $\succ 5$ últimos $=2.79 \%$ \\
\hline Total & 100.00 & Fuente: INEI. \\
\hline
\end{tabular}


últimos departamentos se reparten el $2.8 \%$ del PBI, entre ellos, Huancavelica, Pasco, Apurímac, Tumbes y Madre de Dios; en tanto que los catorce departamentos restantes se reparten el 29.59\% del PBI total.

En relación al mercado laboral, es urgente promover la capacitación de trabajadores para el incremento de la productividad empresarial. La competitividad de la producción nacional se basa en el nivel de la tecnología y en la calidad de la mano de obra ${ }^{11}$.

Otro de los temas vulnerables en nuestra economía es la pobreza. El alto nivel de pobreza existente en el país (54.3\%) y de pobreza extrema $(23 \%)$, resultado de la centralización existente en nuestra economía, es un tema mucho más complejo ya que se encuentra asociado a variables diversas como corrupción, educación y capacitación, distribución del ingreso, investigación y desarrollo.

\section{CONCLUSIONES}

1.Existe un amplio debate sobre las posibilidades de desarrollo de las economías nacionales a partir de la integración o articulación a la producción global, frente a la alternativa de la marginación o exclusión social.

2.Aquellos países que orientan sus esfuerzos a procesos internos de reestructuración de sus economías, destinados a aprovechar las oportunidades y afrontar los retos globales, podrán encontrar una nueva ubicación en la división internacional del trabajo, lo cual implica una respuesta activa frente a los cambios, con acciones de coordinación y promoción emprendidas por el Estado para movilizar a las principales fuerzas productivas de la economía, con el fin de resolver problemas de asignación de recursos que no logran hacer las fuerzas del mercado. Por el contrario, una integración pasiva que implica dejarse llevar por las fuerzas de atracción en un contexto de laissez faire impuesto, conduciría a una creciente subordinación nacional.

3.Uno de los principales objetivos económicos del TLC entre Perú y Estados Unidos es la consolidación y ampliación de los beneficios temporales obtenidos para determinados productos, con la Ley de Preferencias Arancelarias Andinas (ATPA) y su posterior ratificación con la Ley de Promoción Comercial Andina y Erradicación de la Droga (ATPDEA), que vence el 31 de diciembre del presente año.

4.Otro de los objetivos del TLC es intensificar los aspectos fundamentales que orientaron las políticas de ajuste estructural de la globalización neoliberal en las dos últimas décadas, tales como la liberalización, la desregulación y la privatización, dejándose plena libertad a los conductores del proceso de globalización e integración, que son las empresas transnacionales. Estas políticas vienen subordinando los intereses de los estados nacionales a los supranacionales, alentando la alta competencia para atraer inversiones a nuestras economías en base a la desregulación del mercado laboral y al deterioro del medio ambiente.

5.Por lo que el Estado peruano debe propiciar una respuesta a estos procesos, asumiendo una función promotora y coordinadora para una reinserción activa al mercado global, con objetivos concretos en base a un modelo de desarrollo nacional a fin de evitar una mayor polarización de la sociedad.

6.Las asimetrías existentes entre Perú y Estados Unidos podrían acentuarse a medida que avanza el proceso de globalización e integración, si es que nuestra economía responde de una manera pasiva a estos procesos.

7.Las exportaciones peruanas se basan principalmente en recursos naturales debido a que en la economía no se han generado condiciones básicas para una industrialización orientada hacia las exportaciones, lo que hace que la economía peruana sea altamente sensible a las variaciones de precios internacionales de los commodities.

8.Para crecer sostenidamente con miras a satisfacer las necesidades del mercado interno y orientarse al mercado externo, se deben cumplir condiciones vinculadas a la estabilidad macroeconómica, a la productividad y competitividad, lo cual implica superar los cuellos de botella existentes que hacen de la economía altamente vulnerable a los shocks internacionales.

9.Entre las principales barreras para el desarrollo de la competitividad encontramos los aspectos relacionados con la institucionalidad, infraestructura, mercado laboral, descentralización, entre otros. 
10.Uno de los principales temas pendientes en nuestro país es la pobreza. Existe un alto nivel de pobreza (54.3\%) y de pobreza extrema (23\%) que están asociados a variables como centralización, corrupción, educación y capacitación, redistribución del ingreso, investigación y desarrollo.

\section{NOTAS}

1 Gereffi y Korzeniewicz (1994) y Gereffi, (2000), citados en Rivera R., Miguel Angel, (2001) Reinserción, Aprendizaje y Coordinación, Problemas del Desarrollo, vol. 32, num. 127. México, IIEc. UNAM, octubre-dic, pp. 77-78.

2 La flexibilidad de la nueva tecnología permite producir en lotes pequeños una gran variedad de productos, lo que implica que las economías de escala están cediendo su lugar a las economías de alcance, conceptos ampliamente desarrollados en Brown Grossman, Flor, Domínguez Villalobos, Lilia y otros. (1999). Productividad: desafio de la industria mexicana. México, D.F., UNAM, Edit. JUS, pp. 65, 66 y 79.

3 Hobday (1995) y Ernest (1997), citados en Rivera R., Miguel Angel. Op. cit., pp. 80-81.

4 Fajnzylber habla de una articulación de capacidades de innovación y eficiencia a partir de los recursos internos, que permitan propulsar el crecimiento. Fajnzylber, Fernando (1983). La industrialización trunca de América Latina. México, Nueva Imagen, p. 345.

5 La nueva teoría del comercio internacional, en contraposición a la visión ortodoxa de la teoría pura del comercio internacional, sustenta la existencia de rendimientos crecientes en las funciones de producción, lo que trae abajo las bases del Teorema Heckscher-OlhinSamuelson, sustento del modelo actual de industrialización orientado a las exportaciones (IOE). Ver Villarreal, Rene (1989). Economía Internacional. Tomo I, Capítulo 1.

6 Rivera R., Miguel Angel. Op. cit., p. 79

7 Estos conceptos son ampliamente desarrollados en Rivera R., Miguel Angel. Op. cit. pp. 82-84.

8 Dussel, Peters y Liu Xue Dong (2004). Oportunidades y Retos Económicos de China para México y Centroamérica. Naciones Unidas, CEPAL.

9 Araoz Fernández, Mercedes. (2006). Competitividad e integración con la economía mundial. Lima, CIES-CIUP, Edic. Nova Print SAC.

10 Ibid, p. 9.

11 Ruiz, Clemente (1999). Macroeconomía Global, Fundamentos Institucionales y de Organización Industrial. México D.C., UNAM, p. 86.

\section{BIBLIOGRAFÍA}

Araoz Fernández, Mercedes. (2006). Competitividad e integración con la economía mundial. Lima, CIES-CIUP, Edic. Nova Print SAC.
BID (1977). Hacia el libre comercio en el hemisferio occidental, el proceso del ALCA y el apoyo técnico del BID. Documento de Trabajo 218.

Bouby Tolentino, Francisca. (1997). El Tratado de libre comercio y el proyecto de integración económica del Hemisferio Occidental. Revista FCE-UNMSM, Año 2, № 4, julio de 1997.

Brown Grossman, Flor; Domínguez Villalobos, Lilia y otros. (1999). Productividad: desafio de la industria mexicana. México, D.F., UNAM, Edit. Jus.

Dussel, Peters y Liu Xue Dong. (2004). Oportunidades y Retos Económicos de China para México y Centroamérica. Naciones Unidas, CEPAL.

Fajnzylber, Fernando (1983). La industrialización trunca de América Latina, México, Nueva Imagen.

Fundación Konrad Adenauer. (1995). Negociaciones comerciales internacionales: El Perú frente a los bloques de integración económica.

Machlup, Fritz. (1989). A History of thought of economic integration. Columbia University Press.

Robson, Peter. The economics of international integration, Tercera Edición. England, Oxford University Press.

Rivera R., Miguel Angel. (2001). Reinserción, Aprendizaje y Coordinación. México, Revista Problemas del Desarrollo, Vol. 32, №127, IIEc. UNAM.

Ruiz Durán, Clemente. (1999). Macroeconomía Global, Fundamentos Institucionales y de Organización Industrial. México, D.F., UNAM, Edit. Jus, S.A. de C.V.

Villarreal, Rene (1989). Economía Internacional. Tomo I.

Whiting, Van R. Jr. (1993). The Dynamics of Regionalization: road map to an open future?, en The Challenge of Integration: Europe and the Americas, Tniaami FL: the North, South, Center, Chapter 1. 